\title{
An effective inflation treatment for frozen section diagnosis of small-sized lesions of the lung
}

\author{
Zhenzhen Xiang", Jie Zhang", Jikai Zhao, Jinchen Shao, Lanxiang Zhao, Ye Zhang, Gang Qin, Jie Xing, \\ Yuchen Han, Keke Yu \\ Department of Pathology, Shanghai Chest Hospital, Shanghai JiaoTong University, Shanghai 230000, China \\ Contributions: (I) Conception and design: J Zhang, K Yu; (II) Administrative support: K Yu; (III) Provision of study materials or patients: J Zhang; (IV) \\ Collection and assembly of data: Z Xiang, J Zhao, J Shao, L Zhao, Y Zhang, G Qin, J Xing; (V) Data analysis and interpretation: J Zhang, J Zhao, J \\ Shao; (VI) Manuscript writing: All authors; (VII) Final approval of manuscript: All authors. \\ \#These authors contributed equally to this work. \\ Correspondence to: Keke Yu. Room 4B, Building 2, No. 241, West Huaihai Road, Shanghai 230000, China. Email: ykkxx@sina.com.
}

Background: The accuracy of intraoperative pathological diagnosis of small-sized pulmonary nodules including ground-glass opacity (GGO) is important for the surgeon to choose a suitable surgical procedure. Diagnosis of the small-sized lesions of the lung by frozen section (FS) is very difficult for the pathologist because of limited FS technology. Here we tested an effective inflation treatment for FS to improve the diagnostic accuracy of small-sized lung lesions.

Methods: The lung specimens were derived from 113 patients who underwent the surgery at Shanghai Chest Hospital in 2018-2019. The specimens were randomly divided into two groups-uninflated or inflated with diluted embedding medium (Tissue-Tek OCT; Sakura Finetek-USA, CA). The qualities of the FSs were compared with that of corresponding permanent paraffin sections. The FS diagnoses were compared with the final pathologic diagnoses of corresponding permanent sections.

Results: Our results showed that the quality of FS of lung tissue was excellent after inflation with diluted embedding medium (1:1). The total consistency between diagnosis of inflated FS and final pathological diagnosis was $85.7 \%$. In control group, however, the consistency was only $70.2 \%$. When the lesions were less than $1 \mathrm{~cm}$, the consistency between diagnosis of inflated FS and final pathological diagnosis was $90.3 \%$, compared to $64.9 \%$ consistency in uninflated group $(\mathrm{P}=0.014,<0.05)$. When the lesions' computed tomography (CT) measurement threshold $\leq-350 \mathrm{HU}$, the consistency between diagnosis of inflated FS and final pathological diagnosis was $88 \%$ compared to $73.2 \%$ consistency in uninflated group ( $\mathrm{P}=0.071,>0.05$ ). Accuracy, sensitivity and specificity were observed about $90 \%$ for adenocarcinoma in situ (AIS), whereas it is drop to more than $80 \%$ for minimally invasive adenocarcinoma (MIA) in inflated FS.

Conclusions: Inflation with diluted embedding medium (1:1) could make lung tissue expand well during FS. By using this method, small-sized lesions (especially less than $1 \mathrm{~cm}$ ) could be correctly diagnosed to enable adequate surgical procedure, and evaluation of which can be easily based on the intraoperative pathological diagnosis. The small lesions especially AIS could be readily identified on FS. Therefore, this method improves the diagnostic accuracy of FSs for small-sized lung lesions, and has important practical consequences for further therapy.

Keywords: Lung cancer; frozen section diagnosis; ground-glass opacity (GGO); inflation

Submitted Nov 05, 2019. Accepted for publication Jan 18, 2020.

doi: $10.21037 /$ jtd.2020.02.34

View this article at: http://dx.doi.org/10.21037/jtd.2020.02.34 


\section{Introduction}

Lung cancer has become the most common malignancy in the world (1), and it is one of the major causes for cancer death of women in Shanghai in these years. With recent advances in computed tomography (CT) techniques, such as thin-section CT scanning and low-dose CT screening, the detection rate of small-sized lesions increased (2). For stage I lung adenocarcinomas, surgical resection is the standard treatment for patients who can tolerate the procedure. However, the size of resection (i.e., sublobar vs. lobar resection) is a controversial issue (3). Patients with complete resection of adenocarcinoma in situ (AIS) and minimally invasive adenocarcinoma (MIA) have a low risk of recurrence (4-6). Sublobar resection is important for the patients with small sized lung adenocarcinoma. The type of resection is based on pathological diagnosis of frozen section which can be very demanding for small-sized lesions $(7,8)$.

To select the appropriate surgical protocol, accurate histological type is required before or during surgery, especially for patients with lung nodules with diameter $<1 \mathrm{~cm}$. Some research recategorized and compared the diagnostic accuracy of frozen-section and final-pathology according to the latest WHO 2015 classification of pulmonary adenocarcinoma. The overall consistency of diagnosis between frozen section and paraffin section was $80 \%$. The diagnostic error of frozen section mainly occurred in diagnosis of AIS and MIA. The average consistency in diagnosing AIS and MIA was only 64\% (46-74\%) (9-12).

The most common FS error was overdiagnosis of MIA as invasive adenocarcinoma (IA) $(11,12)$. Lepidic pattern was the most important issue that need to be identified on the frozen sections. Tendency diagnosis such as "favor AIS", "favor MIA", or "favor IA" were commonly used in practice. Non-standard diagnosis has hampered surgeons to choose appropriate surgical procedures, therefore, novel methods were needed to improve the diagnostic accuracy of FS. Our research aims to improve the diagnostic accuracy for small-sized lung lesions especially less than $1 \mathrm{~cm}$ by frozen sections. We described here a simple, easy and quick procedure that improves the quality of lung tumor specimens for frozen section.

\section{Methods}

\section{Patients and methods}

\section{Study subjects}

One hundred and thirteen primary patients with smallsized lung lesions, including 35 male and 78 female who had experienced video-associated thoracoscopic surgery (VATS) at Shanghai Chest hospital from 2018 to 2019, were enrolled. The inclusion criteria of cases were: (I) high resolution computed tomography (HRCT) showed pure ground-glass opacity (pGGO) and mixed GGO (mGGO), and the diameter of the lesions was 5-15 mm. (II) Patients were diagnosed as early stage lung adenocarcinoma intraoperatively. Reasons for exclusion were pathologically diagnosed preoperatively with family cancer history or missing consent for our studies. Information on smoking history was obtained by interview with informed consent. Tumor size was measured in fresh specimen. Clinical parameters of study subjects including age, gender, smoking status, tumor size and location, and surgical method of the patients are shown in Table 1. The Ethics Committee of the Shanghai Chest Hospital approved this study. Each resected specimen was carefully examined by the pathologist for any additional findings other than bullae or blebs. CT images and other clinical information are required in each case.

\section{Improved frozen section technique}

To evaluate the effect of embedding medium, we prepared serial dilutions of embedding medium with saline. We tried 3:2 and 2:1 dilution, but they were too sticky to penetrate into lung tissue. And 1:1 diluted embedding medium was suitable for penetration by using 23-gauge needles through the pleura. We gently inflated the specimens from around the nodules and make the lung tissue swelled sufficiently (13). The range of inflated volume $(1-2 \mathrm{~mL})$ depended on the specimen. The lung specimens were randomly divided into two groups-group with uninflated sample and group inflated with embedding medium (1:1). One or two blocks of tumor tissue with the greatest diameter were sampled and two levels section for each block were examined. Each sample was embedded in Cryometrix and frozen at $-20^{\circ} \mathrm{C}$. Samples were cut into 5 - $\mu \mathrm{m}$-thick sections using Lecia-CM30505 cryostat, treated with $95 \%$ alcohol, and subsequently stained with hematoxylin-eosin.

\section{Intraoperative and postoperative diagnosis}

The tumors were diagnosed by pathologists immediately after removed from patients. The frozen section slides for all the cases were independently reviewed by three pathologists. The pathologists were required to determine each case as: AIS, MIA, or IA. The imprecise terms such 
Table 1 Clinicopathologic characteristics of the derivation cohort

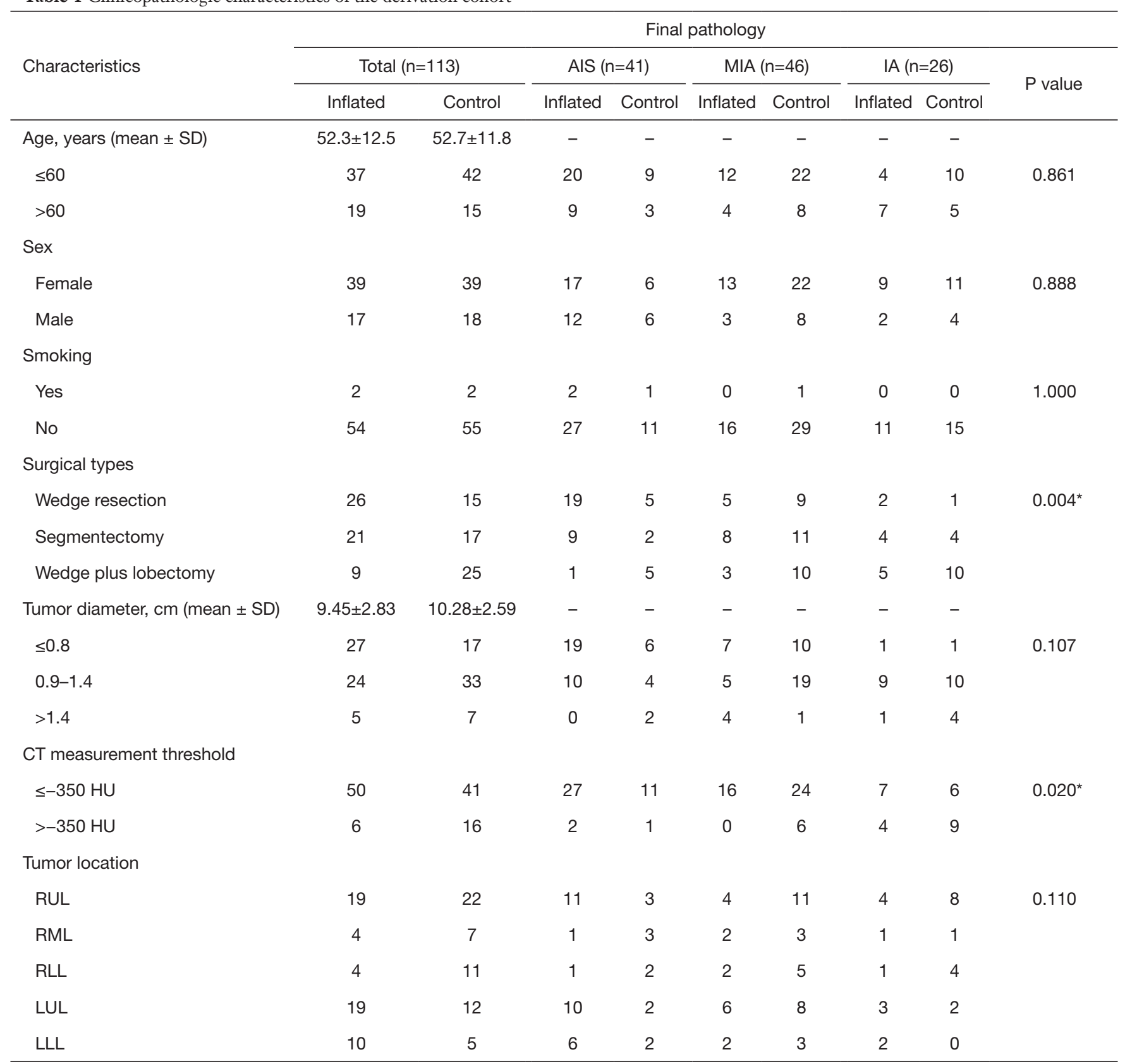

AIS, adenocarcinoma in situ; MIA, minimally invasive adenocarcinoma; IA, invasive adenocarcinoma; SD, standard deviation; RUL, right upper lobe; RML, right middle lobe; RLL, right lower lobe; LUL, left upper lobe; LLL, left lower lobe.

as "favor AIS", "favor MIA", and "favor IA" were not used in this study. After FS diagnosis, the specimens were immersed in the $10 \%$ neutral buffered formalin and embedded in paraffin. All the frozen section diagnosis was compared with final pathologic diagnoses of corresponding permanent paraffin sections. The pathological diagnoses were made according to the 2015 WHO classification for lung adenocarcinoma. All the pathological sections were independently re-evaluated by three senior pathologists (14).

\section{Statistical analysis}

We used Pearson's $\chi^{2}$ test to compare frequencies for categorical variables. Logistic regression was analyzed for 

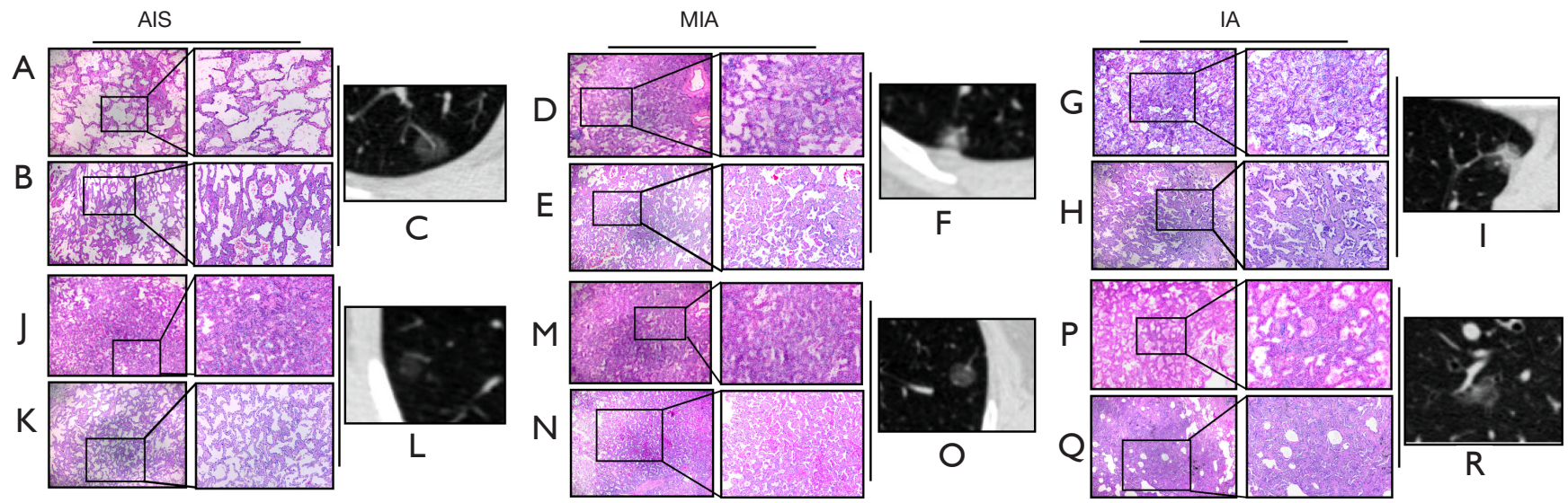

Figure 1 The frozen section, H\&E and CT of the two groups in this study. Original magnifications: left 40x; right 100x. (A) Frozen section of AIS when inflated with diluted embedding medium. Final pathology (B, H\&E) and CT (C) in this case. (D) Frozen section of MIA when inflated with diluted embedding medium. Final pathology (E, H\&E) and CT (F) in this case. (G) Frozen section of IA when inflated with diluted embedding medium. Final pathology (B, H\&E) and CT (C) in this case. (J) Frozen section of AIS in the control group. Final pathology (K, H\&E) and CT (L) in this case. (M) Frozen section of MIA in the control group. Final pathology (N, H\&E) and CT (O) in this case. (P) Frozen section of IA in the control group. Final pathology (Q, H\&E) and CT (R) in this case. CT, computed tomography; AIS, adenocarcinoma in situ; MIA, minimally invasive adenocarcinoma; IA, invasive adenocarcinoma.

predictive factors. SPSS software (version 13.0., SPSS, Inc., Chicago, IL, USA) was used for all statistical analyses. For all tests, $\mathrm{P}<0.05$ indicated a statistically significant difference.

\section{Results}

\section{Clinicopathological features and follow-up}

The research subject with a male: female ratio $1: 2.23$ includes 78 females $(69.0 \%)$ and 35 males $(31.0 \%)$. The youngest patient was 22 years old and the oldest patient was 74 years old. The median age was 52.5 years (Table 1 ). All patients in our study had stage I disease, and were followed up from the day of the lung resection. The followup frequency was every 6 months for 3 years, and then once per year for subsequent years.

\section{Histology}

The appearance of embedding medium-inflated lung tissue was different from that of uninflated lung tissue on frozen section. In the intraoperative frozen sections, cubic or spike-like tumor cells were observed along the alveolar wall. As collapsed alveoli was involved in the hyperplastic interfibrillar substance, so infiltration of tumor cell is difficult to be determined. The representative data in the study were shown in Figure 1. Infusion with 1:1 diluted medium reduced leakage and made specimen expand more completely than the other group. The alveolar walls were straightened, and finer details such as alveolar fenestrations became readily apparent in this group.

\section{Consistency of diagnoses from frozen and paraffin- embedded sections}

The comparison of diagnosis between frozen section and paraffin-embedded section were showed in Table 2. The consistency between the total diagnosis of inflated frozen section and final pathological diagnosis was $85.7 \%(48 / 56)$ whereas it was only $70.2 \%(40 / 57, \mathrm{P}=0.047,<0.05)$ in control group. Then, we subdivided the cases into three groups according to pathological types. When the final pathological diagnosis was AIS, we could see the consistency was $92.9 \%(26 / 28)$ in inflated frozen section compared to only $50.0 \%(12 / 24)$ of consistency in control group $(\mathrm{P}=0.001,<0.05)$. When the diagnoses were MIA and IA, no significant difference was observed between the two groups.

When the lesions were less than $1 \mathrm{~cm}$ in diameter, the consistency between diagnosis of inflated frozen section and final pathological diagnosis was $90.3 \%$ (28/31), while consistency for uninflated group was reduced significantly to only $64.9 \%(24 / 37, \mathrm{P}=0.014,<0.05)$ (Table 3). 
Table 2 Comparison of frozen section and final pathology

\begin{tabular}{lcccc}
\hline \multirow{2}{*}{ Group } & \multicolumn{4}{c}{ Consistency, \% (conformed/cases) } \\
\cline { 2 - 5 } & AIS & MIA & IA & Total \\
\hline Inflate & $92.9(26 / 28)$ & $66.7(14 / 21)$ & $100.0(7 / 7)$ & $85.7(48 / 56)$ \\
Control & $50.0(12 / 24)$ & $76.2(16 / 21)$ & $100.0(12 / 12)$ & $70.2(40 / 57)$ \\
P value & $0.001^{*}$ & 0.495 & 1.000 & $0.047^{*}$ \\
\hline
\end{tabular}

*, P<0.05. AIS, adenocarcinoma in situ; MIA, minimally invasive adenocarcinoma; IA, invasive adenocarcinoma.

Table 3 Comparison of frozen section and final pathology in lesions (less than $1 \mathrm{~cm}$ )

\begin{tabular}{lcccc}
\hline Group & Cases & Conformed number & Consistency \% & P value \\
\hline Inflated & 31 & 28 & 90.3 & $0.014^{\star}$ \\
Control & 37 & 24 & 64.9 \\
\hline
\end{tabular}

${ }^{*}, \mathrm{P}<0.05$.

Table 4 Comparison of frozen section and final pathology in lesions (CT $\leq-350 \mathrm{HU})$

\begin{tabular}{lcccc}
\hline Group & Cases & Conformed number & Consistency \% & P value \\
\hline Inflated & 50 & 44 & 88.0 & 0.071 \\
Control & 41 & 30 & 73.2 & \\
\hline
\end{tabular}

$\mathrm{CT}$, computed tomography.

Table 5 Diagnostic performance of frozen section in two groups

\begin{tabular}{|c|c|c|c|c|c|c|c|c|c|c|}
\hline Category & \multicolumn{2}{|c|}{ Accuracy, \% } & \multicolumn{2}{|c|}{ Sensitivity, \% } & \multicolumn{2}{|c|}{ Specificity, \% } & \multicolumn{2}{|c|}{ PPV, \% } & \multicolumn{2}{|c|}{ NPV, \% } \\
\hline Adenocarcinoma in situ (AIS) & 91.10 & 78.00 & 89.66 & 90.00 & 92.59 & 65.96 & 92.90 & 36.00 & 89.50 & 96.90 \\
\hline $\begin{array}{l}\text { Minimally invasive } \\
\text { adenocarcinoma (MIA) }\end{array}$ & 85.00 & 66.90 & 87.50 & 51.72 & 82.50 & 82.14 & 66.70 & 75.00 & 94.50 & 62.20 \\
\hline
\end{tabular}

PPV, positive predictive value, NPV, negative predictive value.

When the lesions' CT measurement threshold (15) $\leq-350 \mathrm{HU}$, the consistency between diagnosis of inflated frozen section and final pathological diagnosis was $88.0 \%$ (44/50), however, consistency for uninflated group was only slightly reduced to $73.2 \%(30 / 41, \mathrm{P}=0.071,>0.05)$ (Table 4)

Diagnostic performance of frozen section in two groups was shown in Table 5. Accuracy, sensitivity and specificity for AIS in inflated frozen section were about $90 \%$, whereas it is more than $80 \%$ for MIA in inflated frozen section. All the whole indicators were higher in inflated frozen section than in the control group.

\section{Discussion}

Limited surgical resection has been proposed as a treatment for early-stage lung adenocarcinoma $(16,17)$. Identification of patients can be served by sublobectomy depends on the intraoperative pathological diagnosis. As precise final pathological diagnosis has no reference value to the size of resection, the accuracy of frozen section diagnosis is more important than ever been thought. With recent advances in thin-section CT screening, detection rate of smallsized, especially $5-15 \mathrm{~mm}$ nodules increased. However, some researchers reported that the average concordance 
rate of AIS and MIA based on frozen section was only 64\% (46-74\%) (9-12). Intraoperative pathological diagnosis of small-sized lung lesions based on frozen section is dilemmas for pathologists. The main causes for the discrepancy between frozen diagnoses and permanent section diagnoses are sampling error, interpretation error and suboptimal frozen section quality (18). Moreover, it is difficult to identify local alveolar wall collapse in AIS and MIA, and to differentiate invasive components from scar or solid area.

The quality of the frozen section is very important for improving the accuracy of diagnosis of AIS, MIA and lepidic predominant IA on frozen section (19). Here, we reported our experience for improving the accuracy of diagnosis on small-sized lesions in the lung through modifications on inflation during frozen section. The diagnostic concordance rate was significantly improved by our effective inflation treatment. Uninflated lung tissue showed marked artificial atelectasis that mimic hypercellularity such as interstitial cellular infiltration. In contrast, the lung tissue inflated with 1:1 diluted embedding medium showed a much more expanded lung structure as compared to uninflated or saline infused tissue. We used 1:1 diluted embedding medium for penetrating into the lung tissue with 23 -gauge needles through the pleura, as 3:2 and 2:1 diluted media were too sticky to penetrate into lung tissue. In order to prevent the stratification of the solutions, we inflated the tissue immediately after preparation of the solutions. In this study, we evaluated the concordance of frozen section diagnosis and final pathology diagnosis between different pretreatment groups. After inflating with diluted embedding medium, the concordance rate of frozen section and final pathology improved to $85.7 \%$ from the $70.2 \%$ in control group. The concordance rate in our results was slightly lower than previously reported. The reason was that we didn't use the ambigious diagnosis such as "favor AIS", "favor MIA", or "favor IA" in our study. But in other studies, these terms were always involved in calculating concordance rates.

It is of great clinical significance to distinguish between AIS and MIA by frozen-section, because it was particularly useful for surgeons to decide the extent of surgical resection (20). The difficulty in precise frozen section diagnosis was accurate interpretation of MIA. Overdiagnosis of MIA leads to IA, while neglecting of the invasive component leads to AIS. When the noduls were larger than $15 \mathrm{~mm}$, the possibility of IA was high. When the nodulas were smaller than $5 \mathrm{~mm}$, they were often unsuitable for intraoperative diagnosis. So, we focused on patients with lung nodules with diameter $5-15 \mathrm{~mm}$. The conformance rate of diagnosis on tissue sample inflated with diluted embedding medium was $90.3 \%$ compared to only $64.9 \%$ in uninflated group. In the inflated group, the error mainly occurred in MIA. But in the control group, the error mainly occurred in both AIS and MIA. When the lesions' diameter $<1 \mathrm{~cm}$, the difference in sample quality between the two groups was very obvious. According to 2015 WHO classification, diagnosis of MIA mainly focuses on the size of invasion. In frozen section slides, alveolar spaces were frequently collapsed, which could make evaluation of invasion quite problematic. And also, measurement of invasive size can be influenced by fibrosis and scar in adjacent tissue. Additionally, only one FS slice of small-sized lesion was taken for pathological diagnosis from regions that might not be representative of the tumor's pathology; therefore, the invasion status of the whole tumor would be misdiagnosed on the basis of one slide, especially for small-sized lesions. In this study, we improved the quality of lung tumor specimens prepared for frozen section. Embedding medium-inflated lung tissue was demonstrated to be more expanded than uninflated lung tissue. As "false infiltration" in inflated frozen section was not observed, the small-sized lesions could be readily identified in AIS. But in MIA, accurate measurement for invasive size is needed, because of existence of many influencing factors (21). Hence, the diagnosis for MIA was more difficult, which may explain lower accuracy of FS and FP in MIA than in AIS and IA in this study.

We also analyzed the concordance rate between the inflated and uninflated groups when the lesions' CT measurement threshold $\leq-350 \mathrm{HU}$. The concordance rate of sample inflated with diluted embedding medium was $88 \%$, much higher than the control group (73.2\%). Hence it was helpful to read the CT image before sampling for accurate frozen section diagnosis, especially when the lesions' CT measurement threshold $\leq-350 \mathrm{HU}$. It was reported that there was a significant correlation between the GGO lesion and brochioalveolar cell carcinoma in pulmonary small adenocarcinoma $(22,23)$. Our results suggested that diluted embedding medium could improve the quality of frozen section and increase the accuracy of pathological diagnosis.

\section{Conclusions}

In conclusion, our study showed that inflation with diluted embedding medium (1:1) could make lung tissue expand well during frozen section. By employing this method, 
correct diagnosis for small-sized lesions (especially less than $1 \mathrm{~cm}$ ) could be achieved to provide surgeon with more information to evaluate the surgical procedures. The smallsized lesions especially AIS could be readily identified on frozen section. Although the case numbers were limited to draw a definitive conclusion, this method provided a new choice for improving the diagnostic accuracy of frozen sections for small-sized lung lesions.

\section{Acknowledgments}

Funding: This work was supported by the project of Clinical Research Plan of SHDC (No. 16CR3023A).

\section{Footnote}

Conflicts of Interest: All authors have completed the ICMJE uniform disclosure form (available at http://dx.doi. org/10.21037/jtd.2020.02.34). The authors have no conflicts of interest to declare.

Etbical Statement: The authors are accountable for all aspects of the work in ensuring that questions related to the accuracy or integrity of any part of the work are appropriately investigated and resolved. This study was approved by the research institute ethics committee of Shanghai Chest Hospital (Shanghai, China), and the reference number was KS1751. We got their informed consent before the study began.

Open Access Statement: This is an Open Access article distributed in accordance with the Creative Commons Attribution-NonCommercial-NoDerivs 4.0 International License (CC BY-NC-ND 4.0), which permits the noncommercial replication and distribution of the article with the strict proviso that no changes or edits are made and the original work is properly cited (including links to both the formal publication through the relevant DOI and the license). See: https://creativecommons.org/licenses/by-nc-nd/4.0/.

\section{References}

1. Jemal A, Bray F, Center MM, et al. Global cancer statistics. CA Cancer J Clin 2011;61:69-90.

2. National Lung Screening Trial Research Team, Church TR, Black WC, et al. Results of initial low-dose computed tomographic screening for lung cancer. $\mathrm{N}$ Engl J Med 2013;368:1980-91.
3. Liu S, Wang R, Zhang Y, et al. Precise diagnosis of intraoperative frozen section is an effective method to guide resection strategy for peripheral small-sized lung adenocarcinoma. J Clin Oncol 2016;34:307-13.

4. Tsao MS, Marguet S, Le Teuff G, et al. Subtype classification of lung adenocarcinoma predicts benefit from adjuvant chemotherapy in patients undergoing complete resection. J Clin Oncol 2015;33:3439-46.

5. Kent M, Landreneau R, Mandrekar S, et al. Segmentectomy versus wedge resection for non-small cell lung cancer in high-risk operable patients. Ann Thorac Surg 2013;96:1747-54; discussion 1754-5.

6. Russell PA, Wainer Z, Wright GM, et al. Does lung adenocarcinoma subtype predict patient survival?: A clinicopathologic study based on the new international association for the study of lung cancer/american thoracic society/european respiratory society international multidisciplinary lung adenocarcinoma classification. J Thorac Oncol 2011; 6:1496-504.

7. Tsutani Y, Miyata Y, Nakayama H, et al. Appropriate sublobar resection choice for ground glass opacitydominant clinical stage IA lung adenocarcinoma: wedge resection or segmentectomy. Chest 2014;145:66-71.

8. Yano M, Yoshida J, Koike T, et al. The outcomes of a limited resection for non-small cell lung cancer based on differences in pathology. World J Surg 2016;40:2688-97.

9. He P, Yao G, Guan Y, et al. Diagnosis of lung adenocarcinoma in situ and minimally invasive adenocarcinoma from intraoperative frozen sections: an analysis of 136 cases. J Clin Pathol 2016;69:1076-80.

10. Zhu E, Xie H, Dai C, et al. Intraoperatively measured tumor size and frozen section results should be considered jointly to predict the final pathology for lung adenocarcinoma. Mod Pathol 2018;31:1391-9.

11. Walts AE, Marchevsky AM. Root cause analysis of problems in the frozen section diagnosis of in situ, minimally invasive, and invasive adenocarcinoma of the lung. Arch Pathol Lab Med 2012;136:1515-21.

12. Yeh YC, Nitadori J, Kadota K, et al. Using frozen section to identify histologic patterns in stage I lung adenocarcinoma $\leq 3 \mathrm{~cm}$ : accuracy and interobserver agreement. Histopathology 2015;66:922-38.

13. Myung JK, Choe G, Chung D, et al. A simple inflation method for frozen section diagnosis of minute precancerous lesions of the lung. Lung Cancer 2008;59:198-202.

14. Travis WD, Noguchi $M$, Yatabe $Y$, et al. Adenocarcinoma. In: Travis ED, Brambilla E, Burke AP, et al. editors. WHO 
Classification of Tumours of the lung, Pleura, Thymus and Heart. 4th edition. Vol. 7. Lyon: IARC, 2015:26-36.

15. Yanagawa M, Kuriyama K, Kunitomi Y, et al. Onedimensional quantitative evaluation of peripheral lung adenocarcinoma with or without ground-glass opacity on thinsection CT images using profile curves. Br J Radiol 2009;82:532-40.

16. Takahashi Y, Kuroda H, Oya Y, et al. Challenges for realtime intraoperative diagnosis of high risk histology in lung adenocarcinoma: A necessity for sublobar resction. Thorac Cancer 2019;10:1663-8.

17. Dai C, Shen J, Ren Y, et al. Choice of surgical procedure for patients with non-small-cell lung cancer $\leq 1 \mathrm{~cm}$ or 1 to $2 \mathrm{~cm}$ among lobectomy, segmentectomy, and wedge resection: a population-based study. J Clin Oncol 2016;34:3175-82.

18. Trejo Bittar HE, Incharoen P, Althouse AD, et al. Accuracy of the IASLC/ATS/ERS histological subtyping of stage I lung adenocarcinoma on intraoperative frozen sections. Mod Pathol 2015;28:1058-63.

19. Takahashi Y, Ishii G, Aokage K, et al. Distinctive

Cite this article as: Xiang Z, Zhang J, Zhao J, Shao J, Zhao L, Zhang Y, Qin G, Xing J, Han Y, Yu K. An effective inflation treatment for frozen section diagnosis of small-sized lesions of the lung. J Thorac Dis 2020;12(4):1488-1495. doi: 10.21037/ jtd.2020.02.34 histopathological features of lepidic growth predominant node-negative adenocarcinoma $3-5 \mathrm{~cm}$ in size. Lung Cancer 2013;79:118-24.

20. Walts AE, Marchevsky AM. Current evidence does not warrant frozen section evaluation for the presence of tumor spread through alveolar spaces. Arch Pathol Lab Med 2018;142:59-63.

21. Travis WD, Brambilla E, Nicholson AG, et al. The 2015 World Health Organization classification of lung tumors: Impact of genetic, clinical and radiologic advances since the 2004 classification. J Thorac Oncol 2015;10:1243-60.

22. Chae HD, Park CM, Park SJ, et al. Computerized texture analysis of persistent part-solid ground-glass nodules: differentiation of preinvasive lesions from invasive pulmonary adenocarcinomas. Radiology 2014;273:285-93.

23. Lee KH, Goo JM, Park SJ, et al. Correlation between the size of the solid component on thin-section CT and the invasive component on pathology in small lung adenocarcinomas manifesting as ground-glass nodules. J Thorac Oncol 2014;9:74-82. 